\title{
Accurate quantitative wide-field fluorescence microscopy of 3-D spheroids
}

\author{
Elizabeth Leary ${ }^{1,2}$, Claire Rhee ${ }^{1}$, Benjamin Wilks ${ }^{1,2}$, and Jeffrey R. Morgan ${ }^{1,2}$ \\ ${ }^{1}$ Department of Molecular Pharmacology, Physiology and Biotechnology and ${ }^{2}$ Center for Biomedical \\ Engineering, Brown University, Providence, Rl
}

BioTechniques 61:237-247 (November 2016) doi 10.2144/000114472

Keywords: spheroids; quantitative image analysis; live cell imaging; epi-fluorescence; calcein-AM

Supplementary material for this article is available at www.BioTechniques.com/article/114472.

Hundreds of commercially available fluorescent dyes are used to quantify a wide range of biological functions of cells in culture, and their use has been a mainstay of basic research, toxicity testing, and drug discovery. However, nearly all of these dyes have been optimized for use on cells cultured as two-dimensional monolayers. Threedimensional culture systems more accurately recapitulate native tissues, but their size and complexity present a new set of challenges for the use of fluorescent dyes, especially with regards to accurate quantitation. We determined the most accurate method to quantify fluorescence as a function of whether cells were uniformly labeled with dye prior to spheroid formation or if the dye was diffused into the spheroid after its formation. Using multicellular spheroids labeled with calcein-AM via these two different staining methods, we performed time-lapse fluorescence microscopy. For uniformly labeled spheroids, fluorescence was best normalized to volume, whereas for spheroids labeled via dye diffusion, fluorescence was best normalized to surface area. This framework for evaluating dyes can easily be extended to other applications. Utilizing the appropriate size-based normalization strategy enhanced our ability to detect statistically significant differences between experimental conditions.

Three-dimensional (3-D) multicellular spheroids are increasingly being used for research, toxicity testing, and drug discovery (1-3). In general, spheroids more closely mimic the natural biological functions and complexities of native tissues and organs than do cells cultured in 2-D, a property important for phenotypic drug discovery (4-8). Moreover, spheroids and other 3-D technologies are helping to reduce the use of animals in research. For example, some researchers are looking to reduce the number of animal studies by developing cancer-based spheroid models to assist in the drug-screening process $(9,10)$. One commonly used means of assessing these biological functions is the use of fluorescent reporter molecules coupled with quantitative fluorescence microscopy. Fluorescent reporters come in a large range of sizes and can report on a wide scope of diverse biological functions. For example, small fluorescent molecules such as calcein-AM are used to assess cell viability, while genetically engineered fluorescent fusion proteins are used to quantify the expression levels of selected genes. Moreover, fluorescent reporters are either deployed via diffusion into a biological system (e.g., calceinAM) or are embedded in and intrinsic to the biological system (e.g., fluorescent fusion proteins) (11-13). However, nearly all of these fluorescent reporters have been tested in and optimized for use in 2-D monolayer cell culture. Unlike thin monolayers of cells where the $z$-dimension is in the 5- $\mu \mathrm{m}$ range, tissue-engineered 3-D constructs can have a $z$-dimension of 100 $\mu \mathrm{m}$ or greater (14). This increased thickness presents unique challenges for the quanti- tative use of fluorescent reporters, such as the penetration of light and fluorescent molecules into the tissue as well as limitations on the depth of reliable imaging (15-18).

To investigate these issues, we formed an array of 3-D multicellular spheroids on transparent nonadhesive agarose micromolds that could be easily imaged using wide-field fluorescence microscopy (19). We tested the commonly used viability dye calcein-AM as a case study to evaluate how to quantify fluorescence from 3-D spheroids. We added calcein-AM via two different methods: (i) by staining cells prior to self-assembly in order to create uniformly labeled spheroids and (ii) by diffusing dye into the spheroids after self-assembly. To determine the most accurate means of quantifying fluorescence for each scenario, we acquired time-lapse epi-fluorescence

\section{METHOD SUMMARY}

Utilizing calcein-AM, a widely used dye, we investigated the most accurate method for quantification of fluorescence images of 3-D multicellular spheroids. Accurate normalization (using spheroid volume versus surface area) over a range of spheroid sizes depends on how the spheroids were labeled (prestained cells versus diffusion of dye into spheroid). Our method will help investigators avoid significant errors when using fluorescently labeled spheroids. 
images of spheroids of variable sizes, ranging 80-200 $\mu \mathrm{m}$ in diameter. If a spheroid was uniformly stained with calcein-AM, the fluorescence signal was more accurately normalized to the volume of the spheroid regardless of size, whereas if calcein-AM was diffused into the spheroid, the fluorescence signal was more accurately normalized to the surface area of the spheroid. We further validated these findings by testing a total of three cell types (KGN, MCF-7, NHF) and five fluorescent dyes (calcein-AM, calceinred-orange-AM, Hoechst 33342, CellTracker Red CMPTX, CellTracker Green CMFDA). Overall, quantitative fluorescence of spheroids requires optimization and close attention to the method of staining, the range of spheroid sizes, and the duration of spheroid staining. Utilizing the appropriate normalization strategy will reduce significant errors and can enhance the sensitivity and robustness of spheroid-based assays. By improving sensitivity and robustness, spheroid-based assays may become more compatible with high-throughput screening, since fewer spheroids would be needed to discern differences between experimental groups. Furthermore, this method can be applied to numerous other fluorescent dyes to measure a variety of biological functions, thus making our approach applicable to a broad range of basic research fields, as well as toxicity testing and drug discovery.

\section{Materials and methods}

Micro-molded hydrogels, cell culture, and spheroid formation

To create agarose hydrogels, 3-D PetriDish micro-molds (Microtissues, Inc, Providence, RI) consisting of a series of 96 rounded cylindrical pegs atop a rectangular platform were used. Sterile molten UltraPure Agarose (Fisher Scientific, Waltham, MA) (2\% weight/ volume in water) was pipetted into the micromold, allowed to solidify, and released from the micro-mold directly into a 24-well plate. The resulting hydrogel contained a rectangular loading dock on top of a series of 96 round-bottom recesses. Hydrogels were equilibrated with serum-free DMEM supplemented only with 1\% penicillin/streptomycin, degassed to remove air bubbles from recesses, and incubated overnight at $37^{\circ} \mathrm{C}$ with $10 \% \mathrm{CO}_{2}$.

All cell types, including human ovarian granulosa (KGN) cells, breast cancer (MCF-7) cells, and normal human fibroblast (NHF) cells, were grown in DMEM with 10\% fetal bovine serum (FBS) (Fisher Scientific) and
$1 \%$ penicillin/streptomycin at $37^{\circ} \mathrm{C}$ with $10 \%$ $\mathrm{CO}_{2}$. Cells were trypsinized using $0.05 \%$ trypsin, concentrated by centrifugation at $120 \times g$ for $6 \mathrm{~min}$, and counted. Cells were washed once with serum-free DMEM and spun down at $120 \times g$ for 6 min. Cells were re-suspended in serum-free DMEM at one of the following concentrations: $1.33 \times 10^{6}$ cells $/ \mathrm{mL}, 2.67 \times 10^{6}$ cells $/ \mathrm{mL}$, or $4.00 \times$ $10^{6}$ cells $/ \mathrm{mL}$. A $75 \mu \mathrm{L}$-aliquot of each cell suspension was pipetted into the loading dock of each hydrogel to form spheroids of approximately 1000, 2000, or 3000 cells. Cells settled by gravity to the bottom of the micro-wells. After $30 \mathrm{~min}, 1 \mathrm{~mL}$ serumfree DMEM was added per well. Cells were allowed to self-assemble into spheroids for $24 \mathrm{~h}$ prior to performing imaging studies.

Labeling with fluorescent dyes, microscopy, and image analysis

Spheroids were labeled with fluorescent dyes in one of two ways: staining cells as 2-D monolayers prior to spheroid formation or staining the formed 3-D spheroids. Five different fluorescent dyes (all obtained from Life Technologies, Grand Island, NY) were tested: calcein-AM, calcein-RO, Hoechst 33342, CellTracker Green CMFDA (CTG), and CellTracker Red CMPTX (CTR). When staining the cell monolayer, serumcontaining medium was removed from the culture flasks. Fluorescent dyes were reconstituted in serum-free DMEM and incubated with the cell monolayer for $30 \mathrm{~min}$ at $37^{\circ} \mathrm{C}$ with $10 \% \mathrm{CO}_{2}$. All 5 dyes were used to stain monolayers at the following concentrations: $5 \mu \mathrm{M}$ calcein-AM, $5 \mu \mathrm{M}$ CTR, 5 $\mu \mathrm{M}$ CTG, $5 \mu \mathrm{M}$ calcein-RO, and $4 \mu \mathrm{g} / \mathrm{mL}$ Hoechst 33342. After labeling, medium was exchanged with fresh serum-free DMEM, and cell monolayers were incubated for 15 min at $37^{\circ} \mathrm{C}$ with $10 \% \mathrm{CO}_{2}$. After staining, the labeled cell monolayers were trypsinized, counted and seeded into micro-molded gels at various seeding densities. Cells were allowed to self-assemble into spheroids for $24 \mathrm{~h}$ prior to performing imaging studies.

To label formed spheroids by dye diffusion, cell monolayers were trypsinized, counted, and seeded into micro-molded hydrogels at various seeding densities. Unlabeled cells were allowed to self-assemble into spheroids for $24 \mathrm{~h}$ prior to staining with fluorescent dyes and performing imaging studies. Fluorescent dyes were reconstituted in serum-free DMEM, and $1 \mathrm{~mL}$ was added per well. All 5 dyes were used to stain spheroids at the following concentrations: $5 \mu \mathrm{M}$ calcein-AM, $5 \mu \mathrm{M}$ CTR, $5 \mu \mathrm{M}$ CTG, $5 \mu \mathrm{M}$ calcein-RO,
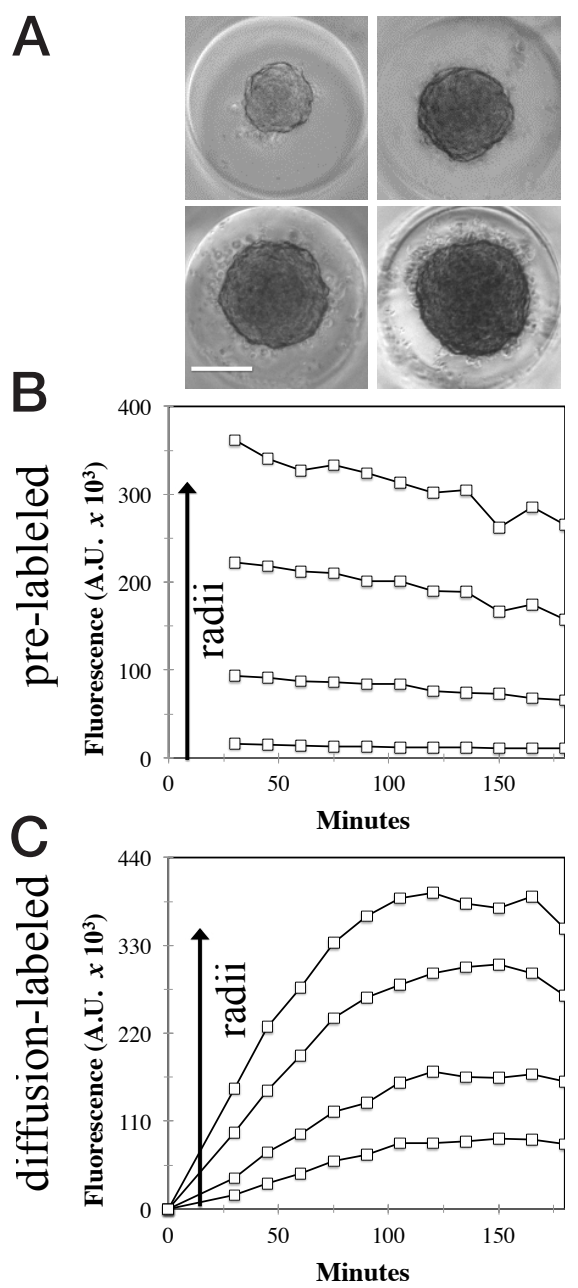

Figure 1. Total fluorescence intensifies with increasing multicellular spheroid radii regardless of whether cells were labeled with calcein-AM in 2-D or 3-D. Spheroids uniformly pre-labeled with $5 \mu \mathrm{M}$ calcein-AM and spheroids labeled with 5 $\mu \mathrm{M}$ calcein-AM by diffusion were prepared by seeding hydrogels at the following seeding densities: 1000, 2000, and 3000 cells/spheroid. Phase contrast images of spheroids of varying sizes were acquired every 15 min over $3 \mathrm{~h}$. Total spheroid fluorescence as a function of time was quantified for individual spheroids formed from pre-labeled cells (B) or spheroids labeled by dye diffusion (C). Each line represents fluorescence data from a single spheroid (radii: $~ 40 \mu \mathrm{m}, 60$ $\mu \mathrm{m}, 80 \mu \mathrm{m}, 100 \mu \mathrm{m})(\mathrm{B}, \mathrm{C})$. Scale bar: $100 \mu \mathrm{m}$.

and $4 \mu \mathrm{g} / \mathrm{mL}$ Hoechst 33342. To determine the optimal concentration of calcein-AM and calcein-RO for diffusion into spheroids, the following concentrations were tested for each dye: $1.25 \mu \mathrm{M}, 2.5 \mu \mathrm{M}$, and $5 \mu \mathrm{M}$.

Conventional inverted microscopy was used to acquire both phase contrast and time-lapse fluorescent images of the spheroids. A Carl Zeiss Axio Vision Observer Z1 equipped with an AxioCam MRm camera (Carl Zeiss Microlmaging, Thornwood, NY), an Xcite 120XL mercury lamp (Exfo Life Sciences Division, Mississauga, Ontario, 
A

Volume
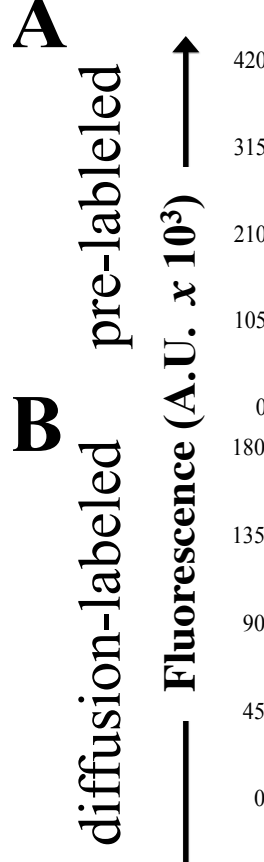

$y=0.03\left(\frac{4}{3} \pi x^{3}\right)$

$135-R^{2}=0.85$

\section{Surface Area}
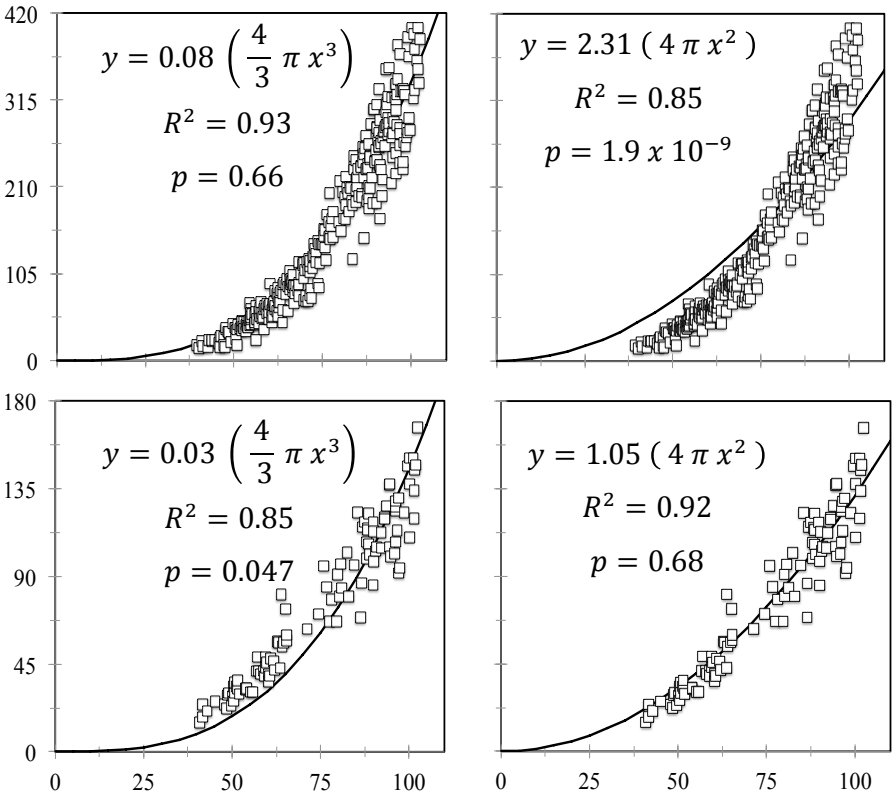

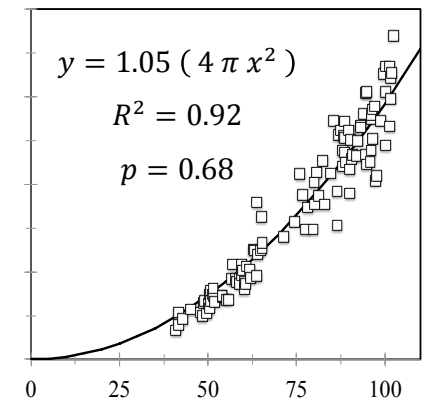

Radii ( $\mu m)$

Canada), and an incubation chamber were used. The following filter configurations were used: excitation and emission cutoffs were $365 \mathrm{~nm}$ and 445/50 nm for Hoechst $33342,545 / 30 \mathrm{~nm}$ and $620 / 60 \mathrm{~nm}$ for CTR and calcein-RO, and $470 / 40 \mathrm{~nm}$ and 525/50 $\mathrm{nm}$ for calcein-AM and CTG. ImageJ was used to measure spheroid length $(x)$, and width $(y)$. Total spheroid fluorescence was calculated by subtracting a backgroundmatched control from the raw spheroid fluorescence for every spheroid at every time point. Spheroid height $(z)$ diameter was calculated to be a ratio of the average $x, y$-diameters (Supplementary Figure S1, Supplementary Materials and Methods). The ratio was determined by using alternative micro-molds containing a single row of spheroids. These molds were imaged using conventional phase contrast microscopy to measure the $x, y$-dimensions and side-view microscopy to measure the $x, z$-dimensions. To determine the relationship between height and length/width, the length and width were averaged and then plotted versus the height for a range of spheroid sizes. Spheroids of KGN cells had a height that was $89 \%$ of the average length and width over a diameter range of 100-270 $\mu \mathrm{m}$ (Supplementary Figure S1D). The average height of spheroids of MCF-7 cells was $84 \%$ of the average length and width over a diameter range of 80-290 um (Supplementary Figure S1E). Instead of assuming that spheroids were perfect spheres, we used these correction factors in our calculations of spheroid shape and fluorescence. Additionally, spheroids $>205$ $\mu \mathrm{m}$ in diameter were excluded from analysis since we have previously shown that their fluorescence signal was no longer linear with respect to size (20). For each labeling method and dye combination, total spheroid fluorescence data from all three seeding densities were pooled and plotted as a function of their radii.

\section{Calculation of prediction curves}

for relating volume/surface

area, radii, and fluorescence

Two different prediction lines were generated to relate the average spheroid radii and fluorescence against either spheroid volume or spheroid surface area. As spheroid radii increased, both spheroid volume and surface area increased, but at different rates. The increase in fluorescence with respect to the radii was measured and compared with the rates of increase in volume and surface area. The prediction lines for volume and surface area were derived from geometric principles, with volume represented as:

$$
V=\frac{4}{3} \pi r^{3}
$$

[Eq. 1]

and surface area represented as:

$$
S A=4 \pi r^{2}
$$

[Eq. 2],
Figure 2. Accurate normalization of calcein-AM fluorescence is dependent on labeling method. Calcein-AM was incorporated into human ovarian granulosa (KGN) spheroids via two different methods. To form pre-labeled spheroids, $5 \mu \mathrm{M}$ calceinAM was added to 2-D monolayers that were then trypsinized and seeded into gels (A). Alternatively, for diffusion-based spheroid labeling, KGN cells were self-assembled into spheroids of varying sizes prior to the addition of $5 \mu \mathrm{M}$ calcein-AM (B). Fluorescence images were acquired $24 \mathrm{~h}$ after self-assembly, and total spheroid fluorescence was plotted as a function of spheroid radii. The spheroid data were plotted against two prediction lines, either a curve of spheroid volume as a function of radii (left column) or a curve of spheroid surface area as a function of radii (right column). Each data point represents a single spheroid. Correlation analysis and Kolmogorov-Smirnov (K-S) tests were performed to determine which prediction line better matched the observed data. When calcein-AM was pre-labeled, the correlation between fluorescence signal and the volume-based prediction line was greater than the correlation to surface area (A). Additionally, the $P$ values for all surface areabased prediction lines were $<0.05$. Alternatively, when calcein-AM was diffused into spheroids, the trends for correlation and K-S tests were reversed, with fluorescence signal better correlating with the surface area-based prediction line (B).

where $r$ is the average of all $x, y$, and $z$ radii of each spheroid. Volume and surface area are units of measurement $\left(\mu m^{3}\right.$ and $\mu m^{2}$, respectively); thus to relate these values to fluorescence arbitrary units (AU), a conversion factor was used. Both volume and surface area conversion factors were derived for each dye at every experimental time point. To derive the conversion factors, fluorescence was plotted with respect to either volume or surface area, and the best-fit line was determined (Supplementary Figure S2). The slope of the best-fit line is the conversion factor that relates fluorescence to either volume $\left(m_{v o l}\right)$ or surface area $\left(m_{s A}\right)$ (Supplementary Figure S2). With the appropriate conversion factors, the prediction lines for increasing volume/surface area with respect to spheroid radii were defined with volume represented as

$$
V=m_{\text {vol }}\left(\frac{4}{3} \pi r^{3}\right)
$$

and surface area represented as

$$
S A=m_{S A}\left(4 \pi r^{2}\right)
$$

[Eq. 4].

Statistical analysis

To determine the relationship between fluorescence signal and spheroid size, both correlation analysis and the 
Kolmogorov-Smirnov test (K-S test) were performed. Correlation between the observed fluorescence signal and either volume-based or surface areabased prediction lines were computed by the following equation:

$$
R^{2}=1-\frac{s s_{\text {res }}}{s s_{\text {tot }}}
$$

where

$$
S S_{\text {res }}=\sum_{i}\left(y_{i}-f_{i}\right)^{2}
$$

and

$$
S S_{\text {tot }}=\sum_{i}\left(y_{i}-\bar{y}\right)^{2}
$$

$y_{i}$ represented observed values, $f_{i}$ represented predicted values, and $\bar{y}$ represented the average observed value. Using MatLab, a 2-sample K-S test at a $5 \%$ significance level was computed between observed fluorescence signal and either the volume-based or surface area-based prediction lines.
When $P<0.05$, we rejected the null hypothesis that both sets of data were derived from the same continuous data set. When $P>0.05$, we failed to reject the null hypothesis.

To determine whether size-based normalization enhances the sensitivity of an assay, fluorescence measurements from four size-matched spheroids per condition were analyzed and plotted three different ways: (i) average and standard deviation (SD) of raw fluorescence values, (ii) normalized to spheroid volume, or (iii) normalized to spheroid surface area, prior to computing the average and SD. A two-tailed Student's t-test was performed with a significance level of 0.05 to compare the experimental conditions. To compare the different methods of normalization, a coefficient of variation (CV) analysis was performed for each of the three methods. Lower Cv values indicate less variation.

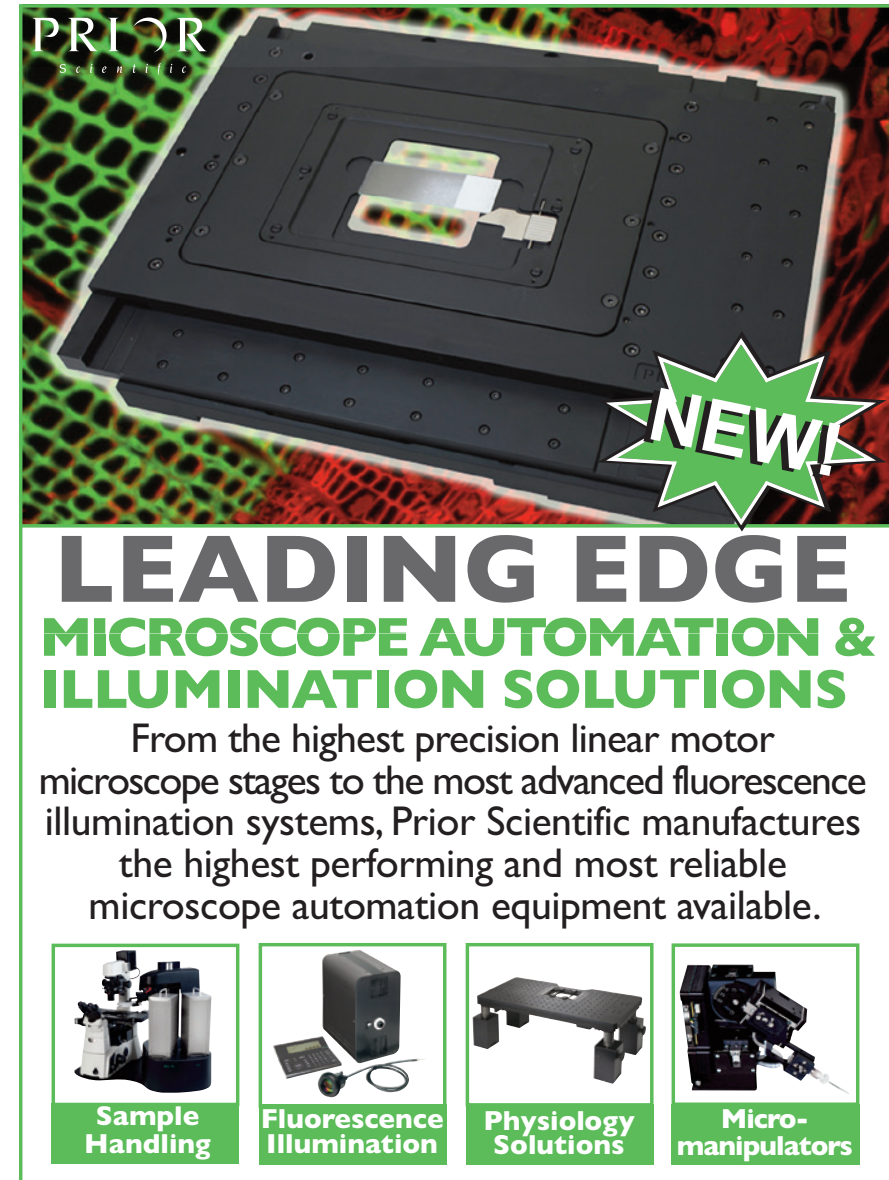

Prior Scientific, Inc. 80 Reservoir Park Dr. Rockland, MA. 02370 Tel: 800-877-2234 Web: www.prior.com

\section{Results and discussion}

An array of multicellular spheroids suitable for time-lapse fluorescence microscopy was formed in micro-molded agarose gels. Spheroid size was controlled by altering the input number of mono-dispersed cells, with higher seeding densities yielding larger spheroids (Figure 1A). To obtain biological information from these spheroids, fluorescent dyes could be used; however, adapting these dyes for use with 3-D spheroids is more complex than for 2-D cell cultures, especially with respect to quantification. In contrast to monolayers of cells where fluorescence signals are typically normalized to either the field of view or the number of cells, spheroids are 3-D multicellular objects that possess unique length, width, and height radii; thus the method of normalization of the fluorescence signal should reflect these variables $(14,21)$. Without accounting for size, larger spheroids yield a greater fluorescence signal, which, if not properly accounted for, could generate inaccurate experimental conclusions. To illustrate the need for a size-based method of normalization of the fluorescence measurements, calcein-AM was incorporated into spheroids by either (i) staining individual cells prior to spheroid self-assembly, or (ii) adding calcein-AM after spheroid selfassembly, thus staining the spheroid by inward diffusion of the dye. The fluorescence signal from pre-labeled spheroids was constant over $3 \mathrm{~h}$ (Figure 1B, Supplementary Figure S3, A and B). In contrast, the fluorescence signal from spheroids labeled by dye diffusion increased over time (Figure 1C, Supplementary Figure S3, C and D). Regardless of how calceinAM was incorporated, increasing spheroid radii yielded an increase in fluorescence (Figure 1, B and C). However, this increase in fluorescence did not imply biological differences but rather the need for a sizebased method to normalize fluorescence.

To eliminate size-based differences in fluorescence, there are two approaches: (i) only analyze spheroids within a narrow range of radii so that size doesn't matter or (ii) normalize signal by an appropriate size-based parameter, which increases the number of analyzable spheroids. We hypothesized that the size-based parameter used to normalize spheroid fluorescence was dependent on the method of dye labeling. When cells were 
Figure 3. Increasing the concentration of calcein-AM diffused into multicellular spheroids improves the fit to surface area. Human ovarian granulosa (KGN) cells were self-assembled into spheroids of varying sizes prior to the addition of calcein-AM at the following concentrations: $1.25 \mu \mathrm{M}(\mathrm{A}), 2.5$ $\mu \mathrm{M}(\mathrm{B})$, and $5 \mu \mathrm{M}(\mathrm{C})$. Fluorescence timelapse images were acquired every 15 min for a total of $3 \mathrm{~h}$ and subsequently analyzed to measure total fluorescence and spheroid radii. The increase in spheroid fluorescence with respect to radii was plotted at various time points after the addition of dye: 30 min (left column), 75 min (middle column), and 135 min (right column). The data were plotted versus a curve of the spheroid surface area as a function of radii. Each point represents a single spheroid. Correlation analysis and Kolmogorov-Smirnov (K-S) tests were performed to determine how the fit to surface area changed in response to altering dose and incubation time. The fit to surface area declined with lower dosages of calceinAM and increased incubation times, as evidenced by lower correlation values. Furthermore, after 135 min of staining with $1.25 \mu \mathrm{M}$ calcein-AM, the K-S test value was $<0.05(A)$.

pre-labeled with dye prior to spheroid formation, we predicted that as spheroid radii increased, total fluorescence would increase at a rate proportional to spheroid
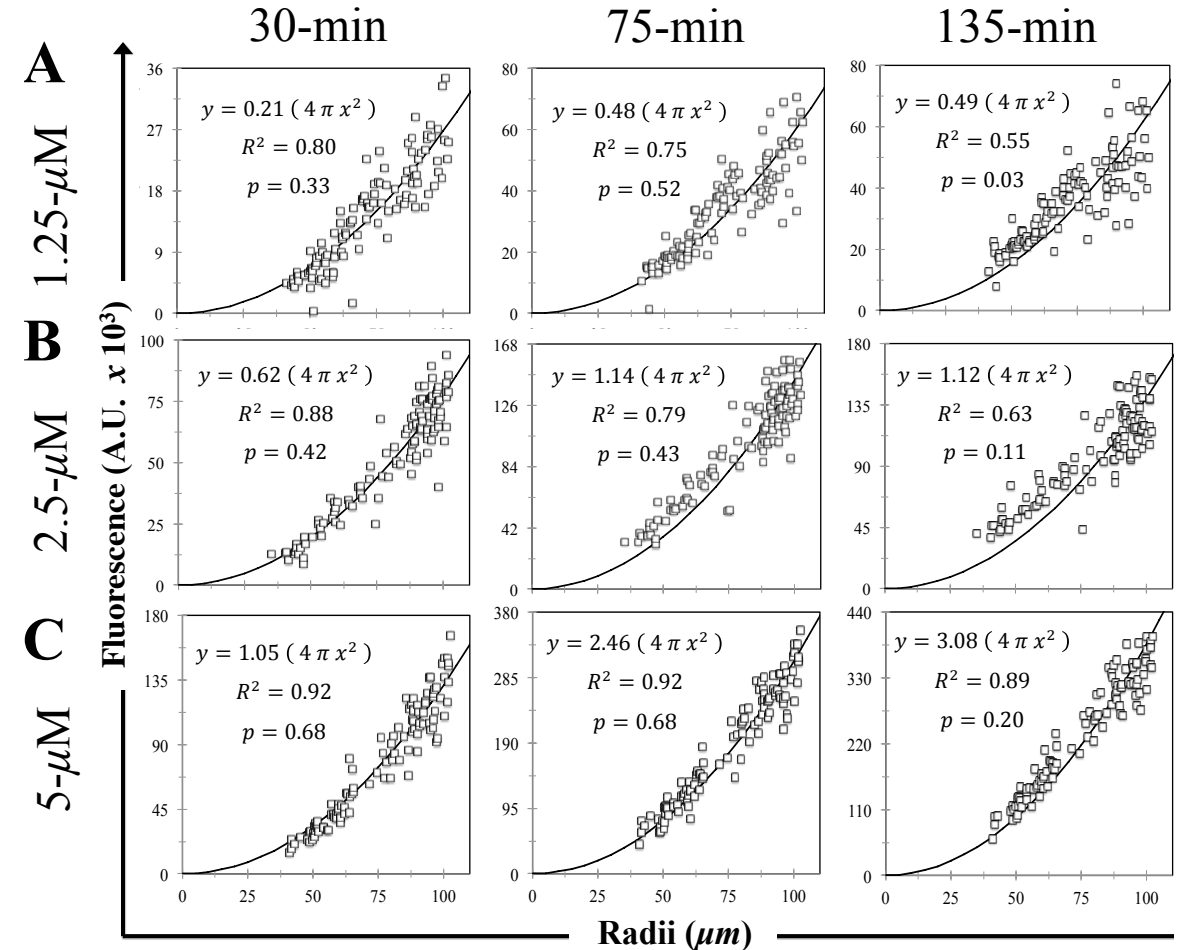

volume since every cell was evenly stained. In contrast, when spheroids were labeled by dye diffusion, we predicted that as spheroid radii increased, total fluores- cence would increase at a rate proportional to spheroid surface area since the amount of dye that could diffuse inwards was dependent upon surface

\section{CLARIOsta}

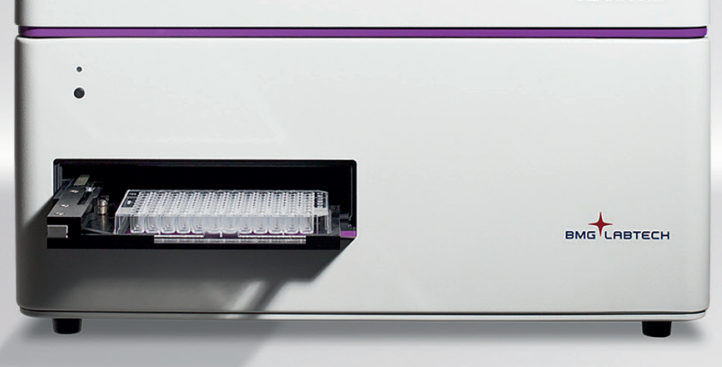

\section{CLARIOstar ${ }^{\circledR}$}

Our revolutionary LVF Monochromator ${ }^{\mathrm{TM}}$ technology combines highest sensitivity and flexibility in a microplate reader.

»The CLARIOstar shows a fantastic performance

with fluorescence, luminescence and UV measurements

We are therefore very satisfied with this device and we strongly recommend it.«

Alfredo Castello, University of Oxford, UK* 
area. Therefore, to determine the best means to quantify spheroid fluorescence, we stained spheroids of varying sizes (diameter: 80-200 $\mu \mathrm{m}$ ) with calceinAM using both labeling techniques. Total spheroid fluorescence was measured and plotted as a function of average spheroid radii. These data were then fitted to a curve of the increase in spheroid volume as a function of increasing spheroid radii or a curve of the increase in surface area as a function of increasing spheroid radii. To determine which curve better predicted the observed increase in fluorescence, correlation analysis and a two-sample K-S test were performed (Figure 2). For spheroids uniformly pre-labeled with calcein-AM, the increase of fluorescence as a function of spheroid radii more closely matched the curve depicting the increase in volume as a function of spheroid radii (Figure 2A). This evidence for a volumebased prediction line was supported both by a greater correlation value and by a $P$ value $>0.05$, with which we failed to reject the null hypothesis that both data sets were derived from a continuous set of data (Figure 2A). This was true for all time points tested over the 3-h time lapse (data not shown). In contrast, for spheroids labeled by calcein-AM diffusion, the increase of fluorescence as a function of increasing spheroid radii more closely matched the curve depicting the increase in surface area as a function of increasing spheroid radii (Figure 2B). Similarly, this match to the surface area-based prediction line was supported by a greater correlation value and by a $P$ value $>0.05$ (Figure 2B). The robustness of this hypothesis was further validated by testing four additional fluorescent dyes (calcein-RO, Hoechst 33342, CTR, and CTG) in a variety of cell types (MCF-7 and NHF) (Supplementary Figures S4-S7). Therefore, we have shown that regardless of differences in excitation/ emission spectra and where the dye resides intracellularly, accurate normalization of the fluorescence measurements was dependent upon how the dye was incorporated into the spheroids. The choice of volume or surface area normalization is important for spheroids because, unlike thin 2-D monolayers where diffusion occurs very rapidly, spheroids are multiple cell-layers thick, presenting a significant barrier to diffusion of fluorescent dyes.

Although other dye characteristics did not seem to affect the effec-
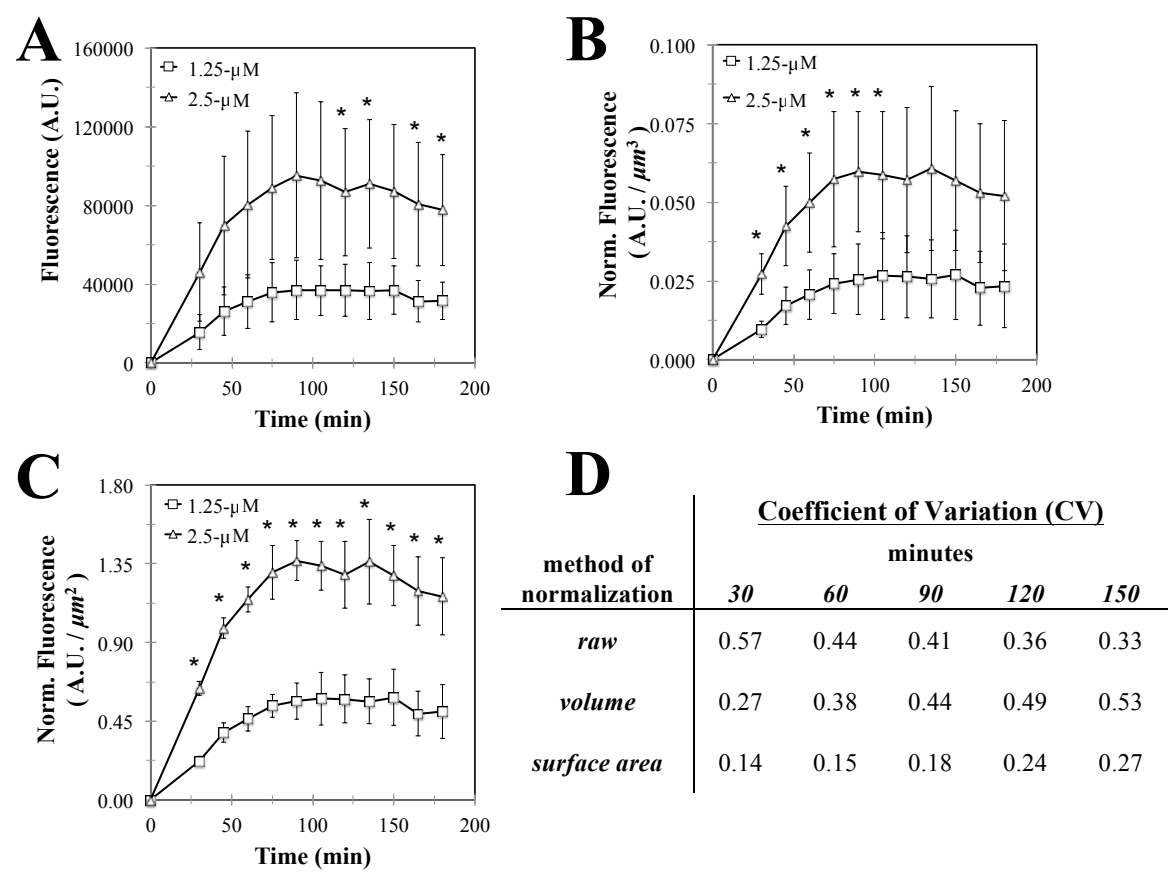

Figure 4. Surface area normalization enhances the ability to detect differences in fluorescence due to different doses of calcein-AM diffusing into multicellular spheroids. Human ovarian granulosa (KGN) cells were self-assembled into spheroids of varying sizes prior to the addition of either 1.25 $\mu \mathrm{M}$ (white squares) or $2.5 \mu \mathrm{M}$ calcein-AM (white triangles). Fluorescence time-lapse images were acquired every 15 min over $3 \mathrm{~h}$ and subsequently analyzed to measure total fluorescence and spheroid radii. Four size-matched spheroids of variable radii (range: 50-95 $\mu \mathrm{m}$ ) were analyzed for each concentration. Diffusion of calcein-AM into 4 size-matched spheroids of variable radii (range: 50-95 $\mu \mathrm{m}$ ) was plotted as a function of time for each concentration. The diffusion of calcein-AM was represented as the mean \pm SD of the spheroids' raw fluorescence $(A)$, volume-normalized fluorescence (B), or surface area-normalized fluorescence (C). A two-tailed Student's $t$-test with a significance level of 0.05 was performed for every time point ("*" indicates statistical significance). Statistically significant differences were observed throughout the entire experiment when fluorescence was normalized by surface area $(C)$, whereas only certain time points were statistically significant when evaluating raw fluorescence (A) or volume-normalized fluorescence (B). The coefficient of variation (CV) was calculated for each method of analysis and for each time point. The CV for surface areanormalized data was lower than that both raw and volume-normalized data for all time points (D).

tiveness of the normalization method, we did determine that for diffusion-based labeling, the concentration of and staining time for calcein-AM was very important. We diffused different concentrations of calcein-AM into spheroids in order to see whether the concentration would alter the fit to the surface area. After forming spheroids of variable size, calcein-AM was diffused into spheroids for $3 \mathrm{~h}$ at the following concentrations: $1.25 \mu \mathrm{M}$, $2.5 \mu \mathrm{M}$, and $5 \mu \mathrm{M}$. For spheroids treated with the lower dye concentrations (1.25 $\mu \mathrm{M}$ and $2.5 \mu \mathrm{M})$, as radii increased, the fit diverged from the surface area prediction line (Figure 3, A and B). This divergence was exacerbated at later time points in the experiment (Figure 3, A and B). Utilizing a higher concentration of calcein-AM ( $5 \mu \mathrm{M})$, the fit to surface area was better matched, and did not diverge as incubation time increased (Figure 3C). The fit to spheroid volume was worse for all time points and concentrations of calcein-AM tested (data not shown). Calcein-RO also exhibited the same trends (Supplementary Figure S8). Overall, we determined that low concentrations and lengthy incubation times reduced the reliability of the model. Therefore, when adapting assays for use in 3-D spheroids, certain optimizations made for 2-D monolayers are not directly transferable and thus further optimization is required.

To highlight the importance of choosing the appropriate size-based normalization method, we measured the fluorescence of spheroids incubated with different calceinAM concentrations (1.25 $\mu \mathrm{M}$ and $2.5 \mu \mathrm{M})$ over 3 h (Figure 4). We plotted the data from 4 size-matched spheroids (radii: 50-95 um) 3 different ways: (i) the average and SD of raw fluorescence values; or the fluorescence of each spheroid normalized by either its (ii) volume or (iii) surface area prior to computing the average and SD. To determine if time points were signifi- 
cantly different, a 2-tailed $t$-test with a significance level of 0.05 was performed. Significant differences between the concentrations throughout the entire experiment were only observed when fluorescence was normalized by spheroid surface area, the appropriate normalization when a fluorescent dye is diffused into a spheroid (Figure 4C). We performed a coefficient of variation (CV) analysis to compare the variability among the three methods of analysis. For all time points tested, the surface area-normalized data had the lowest CV values, indicating the least amount of variation (Figure 4D). Therefore, by utilizing the correct normalization method, we could improve assay sensitivity to discern smaller, yet significant biological differences in experimental conditions. With improved sensitivity, we could analyze fewer spheroids, which will be critical for adapting spheroids for uses such as high-throughput screening.

There are thousands of commercially available fluorescent dyes that probe a diverse range of biological functions. However, these fluorescent dyes have been predominately developed for use with
2-D monolayers only and therefore require careful optimization when used with 3-D spheroids. For example, since spheroids possess a much larger z-dimension than 2-D cell cultures, spheroid height must be taken into consideration when performing imaging studies. Previously, we have shown that only spheroids <205 $\mu \mathrm{m}$ in diameter can be reliably imaged via widefield fluorescence (20). Furthermore, it is widely acknowledged that spheroids significantly $>200 \mu \mathrm{m}$ in diameter have diffusion limitations that can lead to cell death and necrosis in the spheroid core (22). Also, as shown in this study, dye concentrations and incubation times that are well-suited for 2-D monolayers are not necessarily accurate for spheroids and thus require optimization. Fluorescence images of 2-D monolayers are typically normalized to cell number in a fixed field of view, but this method is not applicable to spheroids. For accurate quantitation of fluorescence images of spheroids, it is important to select the normalization method that matches the method of dye staining. Fluorescence images of uniformly labeled spheroids should be normalized by spheroid volume, while fluorescence images of spheroids labeled by inward diffusion of dyes should be normalized by spheroid surface area. Although we tested five fluorescent small molecule dyes, this method of validating and normalizing fluorescence signals from spheroids should be applicable to many other fluorescent dyes and possibly even GFP fusion proteins. However, the different types of GFP reporter assays can be complex (e.g., induction of GFP by a small molecule, diffusion of a GFP encoding virus into a spheroid), so it would be important to perform validation studies and carefully consider the experimental design before choosing a normalization strategy. Furthermore, certain methods of labeling may not be appropriate for every experimental design. For example, forming spheroids from pre-stained cells may not be useful for experiments that extend over multiple days to weeks. Attention to the details of the biological/experimental system is crucial for developing a strategy for image analysis of spheroids. Overall, the correct method of normalization will reduce errors, improve sensitivity, and account for natural variations in spheroid size.

- Endogenously tagged genes developed using CRISPR/Cas9 gene-editing technology

- Suitable for use across a range of applications (WB, FC, ICC, affinity chromatography and more)

\section{with tagged cell lines}

- Increased specificity and simpler quantification

- Antibody validation not required

- Visualize proteins at the physiological level

Ready to order products and services now available 


\section{Author contributions}

E.L. and C.R. designed and performed the series of experiments. B.W. designed ImageJ analysis programs. J.R.M. supervised the project. All authors contributed towards writing the manuscript.

\section{Acknowledgments}

This work was funded in part by seed funds from Unilever, and the generous support of Donna McGraw Weiss '89 and Jason Weiss.

\section{Competing interests}

J.R.M has an equity interest in Microtissues, Inc. This relationship has been reviewed and managed by Brown University in accordance with its conflict of interest policies.

\section{References}

1. Atala, A., F.K. Kasper, and A.G. Mikos. 2012. Engineering complex tissues. Sci. Transl. Med. 4:160rv12.

2. Elliott, N.T. and F. Yuan. 2011. A review of threedimensional in vitro tissue models for drug discovery and transport studies. J. Pharm. Sci. 100:59-74.

3. Tung, Y.C., A.Y. Hsiao, S.G. Allen, Y.S. Torisawa, M. Ho, and S. Takayama. 2011. High-throughput 3D spheroid culture and drug testing using a 384 hanging drop array. Analyst. 136:473-478.

4. Griffith, L.G. and M.A. Swartz. 2006. Capturing complex 3D tissue physiology in vitro. Nat. Rev. Mol. Cell Biol. 7:211-224.

5. Yamada, K.M. and E. Cukierman. 2007. Modeling tissue morphogenesis and cancer in 3D. Cell 130:601-610.

6. Bartosh, T.J., J.H. Ylostalo, A. Mohammadipoor, N. Bazhanov, K. Coble, K. Claypool, R.H. Lee, H. Choi, and D.J. Prockop. 2010. Aggregation of human mesenchymal stromal cells (MSCs) into 3D spheroids enhances their antiinflammatory properties. Proc. Natl. Acad. Sci. USA 107:1372413729.

7. Chang, T.T. and M. Hughes-Fulford. 2009. Monolayer and spheroid culture of human liver hepatocellular carcinoma cell line cells demonstrate distinct global gene expression patterns and functional phenotypes. Tissue Eng. Part A 15:559-567.

8. Vantangoli, M.M., S.J. Madnick, S.M. Huse, P. Weston, and K. Boekelheide. 2015. MCF-7 Human Breast Cancer Cells Form Differentiated Microtissues in Scaffold-Free Hydrogels. PLoS One 10:e0135426.

9. Lemmo, S., E. Atefu, G.D. Luker, and H. Tavana. 2014. Optimization of aqueous biphasic tumor spheroid microtechnology for anti-cancer drug testing in 3D culture. Cell. Mol. Bioeng. 7:344-354.

10. Vinci, M., S. Gowan, F. Boxall, L. Patterson, M. Zimmermann, W. Court, C. Lomas, M. Mendiola, et al. 2012. Advances in establishment and analysis of three-dimensional tumor spheroid- based functional assays for target validation and drug evaluation. BMC Biol. 10.29.

11. Weston, S.A. and C.R. Parish. 1990. New fluorescent dyes for lymphocyte migration studies. Analysis by flow cytometry and fluorescence microscopy. J. Immunol. Methods 133:87-97.

12. Chalfie, M., Y. Tu, G. Euskirchen, W.W. Ward, and D.C. Prasher. 1994. Green fluorescent protein as a marker for gene expression. Science 263:802805.

13. Singhvi, R., A. Kumar, G.P. Lopez, G.N. Stephanopoulos, D.I. Wang, G.M. Whitesides, and D.E. Ingber. 1994. Engineering cell shape and function. Science 264:696-698.

14. Pampaloni, F., E.G. Reynaud, and E.H. Stelzer. 2007. The third dimension bridges the gap between cell culture and live tissue. Nat. Rev. Mol. Cell Biol. 8:839-845.

15. Pampaloni, F., N. Ansari, and E.H. Stelzer. 2013. High-resolution deep imaging of live cellular spheroids with light-sheet-based fluorescence microscopy. Cell Tissue Res. 352:161-177.

16. Boutin, M.E. and D. Hoffman-Kim. 2015. Application and assessment of optical clearing methods for imaging of tissue-engineered neural stem cell spheres. Tissue Eng. Part C Methods 21:292-302.

17. Robertson, F.M., M.A. Ogasawara, Z. Ye, K. Chu, R. Pickei, B.G. Debeb, W.A. Woodward, W.N. Hittelman, et al. 2010. Imaging and analysis of 3D tumor spheroids enriched for a cancer stem cell phenotype. J. Biomol. Screen. 15:820-829.
18. Schneckenburger, H., P. Weber, M. Wagner, S. Schickinger, V. Richter, T. Bruns, W.S. Strauss, and R. Wittig. 2012. Light exposure and cell viability in fluorescence microscopy. J. Microsc. 245:311-318.

19. Napolitano, A.P., D.M. Dean, A.J. Man, J. Youssef, D.N. Ho, A.P. Rago, M.P. Lech, and J.R. Morgan. 2007. Scaffold-free three-dimensional cell culture utilizing micromolded nonadhesive hydrogels. Biotechniques 43:494-500.

20. Achilli, T.M., S. McCalla, J. Meyer, A. Tripathi, and J.R. Morgan. 2014. Multilayer spheroids to quantify drug uptake and diffusion in 3D. Mol. Pharm. 11:2071-2081.

21. Smith, L.E., R. Smallwood, and S. Macneil. 2010. A comparison of imaging methodologies for $3 \mathrm{D}$ tissue engineering. Microsc. Res. Tech. 73:11231133.

22. Casciari, J.J., S.V. Sotirchos, and R.M Sutherland. 1988. Glucose diffusivity in multicellular tumor spheroids. Cancer Res. 48:3905-3909.

Received 01 July 2016; accepted 22 September 2016.

Address correspondence to Jeffrey R. Morgan, Brown University, G-B 393, Biomed Center, 171 Meeting St., Providence, RI 02912. E-mail: Jeffrey_ Morgan@Brown.edu

To purchase reprints of this article, contact: biotechniques@fosterprinting.com

\section{DETECT RRISPR GENE EDITING EVENTS Using Agarose Gels}

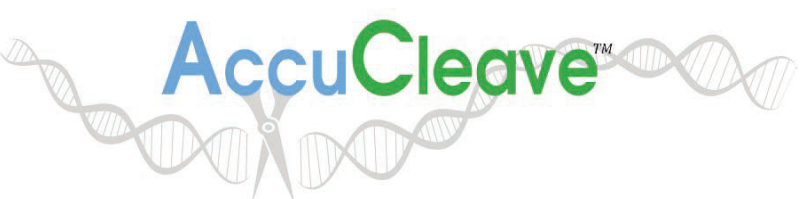

Streamline gene editing workflows with a simple and accurate enzymatic assay.

\section{AccuCleave $^{\mathrm{TM}}$ T7 Kit}

- T7 based enzyme kit for mutation frequency detection of CRISPR events.

\section{AccuCleave $^{\mathrm{TM}}$ C2 Control DNA Kit}

- Contains 2 Control DNA fragments - intact and -2 deletion fragment.

\section{AccuCleave $^{\mathrm{TM}}$ CI5 Control DNA Kit}

- Contains 15 Control DNA fragments - intact, $\pm 1, \pm 2, \pm 10 \mathrm{InDels}$, and 8 point mutations.

\section{Start now at} www.aati-us.com/AccuCleave

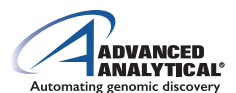

